[0212-7199 (2006) 23: 6; pp 260-263] ANALES DE MEDICINA INTERNA Copyright $\odot 2006$ ARAN EDICIONES, S.L.

AN. MED. INTERNA (Madrid) Vol. 23 , N. ${ }^{\circ}$ 6, pp. 260-263, 2006

\title{
Serología hepatitis C: estudio de prevalencia en población post mortem
}

\author{
A. ARROYO FERNÁNDEZ, M. T. CARBONÉ COTÉ
}

Instituto de Medicina Legal de Cataluña. Barcelona

HEPATITIS C SEROLOGY: PREVALENCE STUDY IN POSTMORTEM POBLATION

\section{RESUMEN}

Objetivos: estudiar la prevalencia de la infección por VHC en una población cadáver de la ciudad de Barcelona a la que se le practica la necropsia judicial. Comparar los datos con los conocidos en epidemiología y salud pública.

Diseño: estudio observacional analítico, retrospectivo llevado a cabo en el Laboratorio del Instituto de Medicina Legal de Cataluña, en 169 cadáveres en los que se solicitó la determinación de VHC por el médico forense, durante el año 2004.

Metodología: análisis mediante técnica de enzimoinmunoensayo para detección de anticuerpos VHC en el analizador AsXym (Abbot).

Variables principales: se incluyeron las variables edad, sexo y causa de muerte dividida en dos grupos, muerte natural y muerte por reacción adversa a drogas. La variable de respuesta fue hepatits $\mathrm{C}$ positivo o negativo.

Resultados: la prevalencia del resultado positivo a la infección en el grupo de muertes naturales fue del 10,8\%. En el grupo de muerte por reacción adversa a drogas fue del $60 \%$.

Conclusiones: La prevalencia encontrada es mayor que la descrita en la literatura especialmente en el grupo de muertes naturales. Se considera que el virus afecta a un número de población que se halla asintomática. La realización de estudios periódicos de prevalencia de esta infección tienen interés en epidemiología y salud pública.

PALABRAS CLAVE: Hepatitis C. Medicina forense. Prevalencia hepatitis $\mathrm{C}$ post mortem.

\section{ABSTRACT}

Aims: to assess infection HCV prevalence in corpses where judicial necropsy was ordered by court, in Barcelona city. To compare results with those actually known in epidemiology and public health services.

Material and Methods: retrospective, observational analitic study carried out in the department laboratory of Medico Legal Institute, in Catalunya (Spain).

The sample was proceeding from 169 corpses where HCV analysis was solicitated by the forensic doctors, during 2004.

Methods: analytical procedure followed was enzimoinmune essay to detect HIV antibodies, using AsXym analyzer (Abbott).

Variables included were sex, age and cause of death. Cause of dead was divided in two groups: natural death and drugs relationated death. Results of the analysis were classified as $\mathrm{HCV}+$ and $\mathrm{HCV}$ - according to laboratory test specifications.

Results: HCV prevalence was 10,8\% in natural deaths group and $60 \%$ in drug relationated death group.

Conclussions: HCV prevalence described is higher than reported in published studies, specially regarding to natural deaths group. Nowadays virus affecting to asymptomatic people is considered. Periodic HCV prevalence studies in general poblation must be carried out to provide dades to epidemiology and health public services.

KEY WORDS: Hepatitis C. Forensic sciences. Hepatitis C prevalence. Post mortem analysis.

Arroyo Fernández A, Carboné Coté MT. Serología hepatitis C: estudio de prevalencia en población post mortem. An Med Interna (Madrid) 2006; 23: 260-263.

\section{INTRODUCCIÓN}

El virus de la hepatitis $\mathrm{C}$ es una preocupación sanitaria emergente a nivel mundial con una población afectada y con riesgo de desarrollar cancer de hígado, cirrosis o fracaso hepático. Los modos de transmisión incluyen el uso intravenoso de drogas, los derivados sanguíneos, los tatuajes y las relaciones sexuales, este último mecanismo con mucha menor importan- cia. El mayor factor de riesgo para adquirir la infección es la via intravenosa. En los últimos años se ha evaluado el riesgo por medio de transfusiones, aunque éste ha disminuido desde que los fluidos se someten a las pruebas de detección previas (1).

Los estudios basados en la detección de anticuerpos anti VHC estiman que el $1 \%$ de la población mundial, se infecta con el virus, con distintas variaciones geográficas. Así en

Trabajo aceptado: 4 de enero de 2006 
Norteamérica es de $1,1 \%$, en América Central de 0,4\% y en Sudamérica de 2,6 a 2,9\% (2). En España se estima que esta cifra es algo inferior al 3\% (3).

Actualmente se considera que puede haber hasta un $75 \%$ de la población que se halla contagiada por el virus, pero no conoce la infección y se halla asintomática. En el periodo de 5 a 10 años estos pacientes pueden desarrollar las enfermedades derivadas de la misma (4).

El objetivo e este trabajo ha sido estudiar la prevalencia de la infección por VHC, mediante métodos serológicos de detección de anticuerpos, en una población cadáver de la ciudad de Barcelona a la que se le practica la necropsia. Comparar estos datos con los actualmente conocidos en epidemiología y salud pública.

\section{MATERIAL Y MÉTODOS}

Estudio observacional analítico retrospectivo llevado a cabo en el Servicio de Laboratorio del Instituto de Medicina Legal de Cataluña.

Muestra. Cadáveres que ingresaron para la realización de la necropsia judicial en el Servicio de Patología del Instituto de Medicina Legal de la ciudad de Barcelona. Esta muestra comprende cadáveres procedentes de muertes violentas y de muertes súbitas naturales cuyas causas de defunción no habían sido certificadas por los facultativos.

Tamaño de la muestra. Ciento sesenta y nueve cadáveres, en los que se solicitó la determinación de VHC por el médico forense durante el año 2004.

Criterios de exclusión. Cadáveres donde había transcurrido más de 48 horas de la muerte hasta la realización de la necropsia.

Metodología. La sangre se extrajo del sistema venoso y se conservó a $4^{\circ}$ hasta su procesamiento. Se centrifugó 3 veces a $10.900 \mathrm{rpm}$ a fin de separar el suero. Las muestras se procesaron en el analizador AsXym (Abott) mediante técnica de enzimoinmunoensayo para detección de anticuerpos VHC, procedimiento MEIA para analitos de alto peso molecular (HCV versión 3.0). Los resultados se informaron como positivos y negativos, según los valores de puntos de corte que el fabricante marca en sus especificaciones.

Variables de estudio y recogida de datos. En el estudio se incluyeron como variables categóricas el sexo, la causa de muerte y el resultado del test VHC, así como la variable cuantitativa edad, a su vez categorizada en niveles. A las variables cualitativas se les asignaron los valores: sexo ( $1=$ masculino, $2=$ femenino), causa de muerte $(1=$ natural, 2 = reacción adversa a drogas), resultado del análisis VHC $(1=$ positivo, $2=$ negativo $)$ y edad categorizada en grupos $(1=$ menor de 30 años, $2=$ entre 30-55 años, 3= mayor de 55 años). Los resultados se analizaron mediante el programa SPSS. 11 para Windows mediante pruebas de asociación de chi cuadrado.

Se describieron las frecuencias de las variables cualitativas, la media y desviación estándar de la variable cuantitativa edad y se comparó la asociación de la variable en estudio, hepatitis $\mathrm{C}$, con el sexo, la edad en grupos y la causa de muerte.

Dentro de la variable causa de muerte, dividida en dos grupos, natural y reacción adversa a drogas, la asignación de muerte natural incluyó también otras muertes no naturales o violentas, ya que éstos otros tipos de muerte no afectaban a la finalidad del estudio al no ser factores de riesgo para la infección del VHC.

\section{RESULTADOS}

La edad media de los cadáveres fue de 49,4 años, con una DE de 19,6 y rango de 80 (máximo 93, mínimo 13). Los resultados de prevalencia, frecuencias y asociación de variables se exponen en las Tablas I, II, y III y en los figuras I, II y III.

TABLA I

FRECUENCIAS DE LAS VARIABLES EN LA POBLACIÓN TOTAL $(\mathrm{N}=169)$

\begin{tabular}{lcc}
\hline & Número de sujetos & \% de sujetos \\
\hline VCH positivo & 33 & 19,5 \\
VCH negativo & 136 & 80,5 \\
Muerte natural & 139 & 82,5 \\
Muerte reacción adversa a drogas & 30 & 17,8 \\
Sexo masculino & 136 & 80,5 \\
Sexo femenino & 33 & 19,5 \\
Edad < 30 años & 32 & 18,9 \\
Edad entre 30-55 años & 78 & 46,2 \\
Edad $>$ 55 años & 59 & 34,9 \\
\hline
\end{tabular}

TABLA II

PREVALENCIA SEROPOSITIVIDAD HEPATITIS C

\begin{tabular}{lc}
\hline Población total & $19,5 \%$ \\
Población muerte natural & $10,7 \%$ \\
Población muerte reacción adversa a drogas & $60 \%$ \\
\hline
\end{tabular}

TABLA III

ASOCIACIÓN SEROLOGÍA HEPATITIS C Y VARIABLES ESTUDIO

\begin{tabular}{lrc}
\hline VHC positivo-sexo & 0,500 & 0,48 \\
VHC positivo-causa de muerte & 38,024 & 0,000 \\
VHC positivo-edad en grupos & 6,015 & 0,049 \\
\hline
\end{tabular}

\section{DISCUSIÓN}

Nuestros resultados sobre la prevalencia del VHC, basada en la detección de anticuerpos, en la población de estudio no coinciden con muchos de los publicados en la literatura especializada, siendo sensiblemente superior. Si consideramos el primer grupo, causa de muerte natural, la prevalencia es de $10,7 \%$. El objetivo principal de nuestro estudio era constatar este valor y evaluar el posible aumento o disminución del mismo en nuestra comunidad urbana. En el segundo grupo, causa de muerte reacción adversa a drogas, donde se halla el principal factor de riesgo consumo de drogas por vía intravenosa, la prevalencia es del $60 \%$. Por último, estimada la prevalencia global en el conjunto de los dos grupos es de 19,5\%. Está descrito que los usuarios de drogas tienen un $20 \%$ más de seropositividad a lo estimado en la población general (5). 


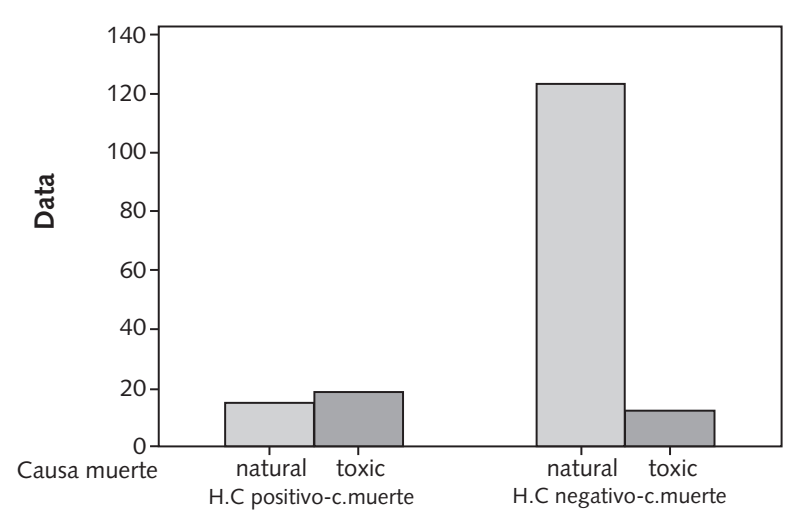

Fig. 1. Chart of H.C positivo-C.muerte; H.C negativo-c.muerte vs. Causa muerte.

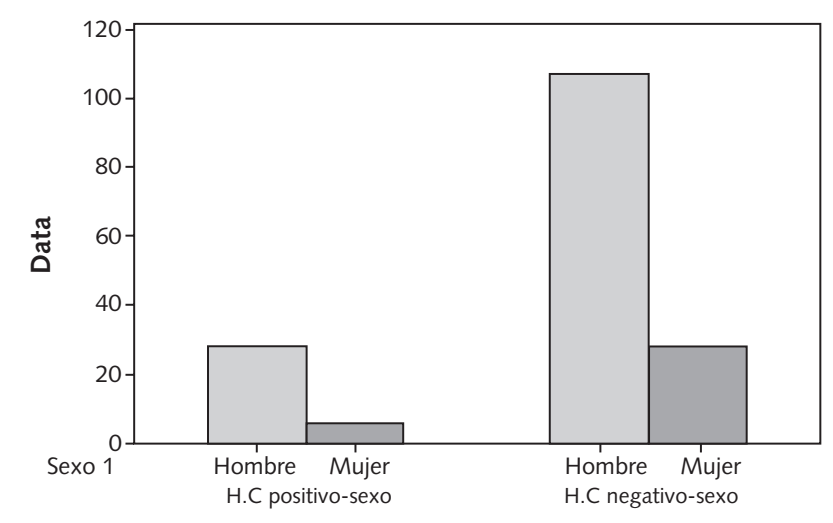

Fig. 2. Chart of H.C positivo-sexo; H.C negativo-sexo vs. Sexo 1.

En nuestro estudio la asociación de la seropositividad con el consumo de drogas es claramente significativa $(p=0,000)$. La asociación con la edad categorizada indica que es más frecuente en el rango de edad entre 30-55 años ( $p=0,049)$. No obstante en este grupo se encuentra la mayoría de sujetos cuya causa de muerte es la reacción adversa a drogas, por lo que esta asociación se podría hallar sobreestimada. No hemos hallado diferencias significativas entre la positividad a VHC y el sexo $(p=0,469)$.

En muchos estudios de prevalencia de infección por VHC se indica la relación entre diferentes orígenes del contagio, no solamente el abuso de drogas sino las transfusiones de sangre y otras circunstancias como el encarcelamiento y las malas condiciones higiénico sanitarias (6).

En nuestro país, comparando con otras zonas, en un estudio realizado en el norte de España la prevalencia fue de $1,6 \%$. Se evaluaban los factores de riesgo de drogadicción, transfusiones previas o sometimiento a cirugía, con mayor proporción de afectados entre los jovenes en el caso de la drogadicción y la situación de cirugía previa o transfusiones en los pacientes de más edad (7). Otro estudio poblacional en la Rioja, en el año 1988, aporta unos datos de prevalencia globales del $2 \%(8)$.

En diferentes paises se sigue la evolución de la infección del virus $\mathrm{VHC}$, en distintos colectivos y áreas geográficas.

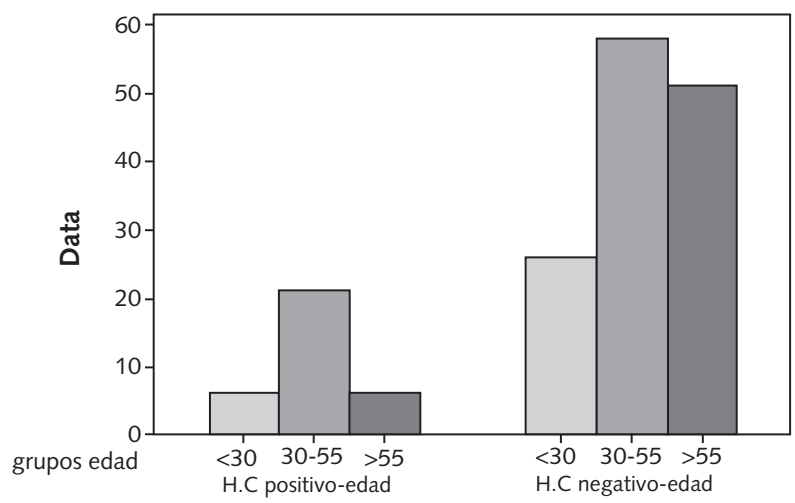

Fig. 3. Chart of H.C positivo-edad; H.C negativo-edad vs. grupos edad.

Algunos estudios sectorizados, como en un grupo de donantes de sangre llevado a cabo en Arabia Saudí, indican una prevalencia del virus de $0,4 \%$ (9) y de 0,6 a $2,2 \%$ se describe en otro colectivo de donantes de sangre en Cuba (10). En paises de mayor nivel económico como en Noruega la prevalencia global de la infección fue de $0,7 \%$. En este estudio los mecanismos de contagio de adicción a drogas suponían el $67 \%$, las transfusiones en el $6 \%$ y origen desconocido en el $27 \%$. Este valor es inferior al hallado en otros países o medios. En este trabajo también se consideraron factores educacionales y sociales dentro de la población (11).

El contagio entre usuarios de drogas está bien documentado. Sherrif describió una prevalencia estimada de $26 \%$ en un grupo de 98 sujetos en una población marginal y sin hogar en la ciudad de Oxford. Entre ellos el mayor factor de riesgo era asimismo el consumo de drogas, a lo que se unía la pobreza, y el compartir objetos de uso común en el ambiente de la drogadicción (12). En nuestro análisis no hemos podido incluir los antecedentes y origen del contagio en los sujetos seropositivos, al ser un estudio retrospectivo, no constar datos y no considerarse fiable la información recibida.

Asimismo se ha estudiado la prevalencia de la infección según distintas razas o la influencia en población afecta de ciertas patologías como el trastorno mental. Butterfield y cols., en un grupo de veteranos de guerra encuentra una prevalencia de VHC de 18,9\%. En relación con la raza describe mayor número de afectados en sujetos afroamericanos que en caucasianos, 21,3 versus $15,5 \%$, donde el factor de la drogadicción sigue teniendo más injerencia que la raza (13). Otro estudio en este sentido encuentra seropositividad al virus en un $7-8 \%$ en la población de raza negra o hispana (14). En nuestro estudio no se incluyeron sujetos de otras razas o etnias siendo una población homogénea.

En colectivos con otras patologías como la diabetes también se hallan cifras de alta prevalencia $11,55 \%$ sin que se detectara un factor epidemiológico específico para la infección (15).

La conclusión más importante de nuestro estudio es que la prevalencia de la seropositividad al VHC en el conjunto de la población cadáver estudiada en la ciudad de Barcelona es alta. Las limitaciones del estudio son que no se han hecho pruebas virológicas de determinación del genoma o carga viral ni del antígeno Core o serotipos de VHC, ni se han evaluado los 
valores predictivos. No obstante, algunos estudios de prevalencia se basan en esta metodología de detección de anticuerpos y su utilidad post mórtem también ha sido investigada y publicada $(16,17)$.

No creemos que el tratarse de población cadáver introduzca sesgos en las cifras halladas. Es posible que ésto ocurra sólo en el grupo de los sujetos fallecidos adictos a drogas donde el mayor deterioro físico o un alto grado de adicción desencadenaran la muerte. Las altas cifras de prevalencia halladas no se han comparado versus un colectivo global de adictos a drogas donde la prevalencia ya descrita en la literatura es $20 \%$ superior a la generalidad, al no ser objetivo de nuestro trabajo. Nuestros resultados, un $60 \%$ de prevalencia en población que fallece por reacción adversa a drogas es más cercano al $75,8 \%$ hallado por Siragusa y cols. en un estudio postmortem de 793 heroinómanos (18). No obstante la prevalencia del VHC en el grupo sin factores de riesgo conocidos, el de las muertes naturales, que es el principal objetivo de nuestro estudio, es asimismo más elevada que las descritas en la literatura.

En la actualidad se considera que hay un descenso de nuevas infecciones o se mantienen estables pero la preva- lencia de enfermedades hepáticas relacionadas con la infección VIH aumenta (19) y en términos de coste y mortalidad se espera que aumenten los valores en las próximas decadas (20).

El control actual de las donaciones y las medidas informativas sobre el uso de material en consumidores de drogas así como la información sanitaria sobre contagios por vía sexual deben hacer que estas cifras vayan disminuyendo y que las políticas sanitarias incluyan la detección de anticuerpos como pruebas rutinarias que permitan identificar a los pacientes. Otro objetivo a seguir es disminuir el contagio intrafamiliar en los sujetos con infección VHC asintomática, al no existir una vacunación adecuada.

La realización de estudios periódicos de prevalencia de infección por VIH tienen interés en epidemiología y salud pública. Las variaciones en el tiempo de estos valores permiten valorar la evolución de estos marcadores y relacionarlos con hábitos y costumbres, así como diseñar campañas de prevención e higiénico sanitarias para impedir la extensión de la infección y sus complicaciones posteriores (21) o iniciar tratamientos en los casos que proceda.

\section{Bibliografía}

1. Disponible en htt//db.doyma.es/:Jano-on -line 5-2-2004.

2. Beltran M. Artículo de Revisión. Repertorio de Medicina y Cirugía 2002; (11); 1 .

3. Puigdengolas X, Sánchez JM. Hepatitis agudas. En: Gastroenterología y Hepatología en Atención Primaria. Aula Médica Madrid 2002: 452.

4. Disponible en htt//db.doyma.es/: Jano -on -line 29-1-2003.

5. Pereiro C, castro A. Hepatitis por virus $\mathrm{C}$ y drogadicción. Socidrogalcohol 2004; (16). 3

6. Ouzan D, Cavailler P, Hofliger P, Mamino C, July H, Trau A. Modalities of care in antiHCV positive patients identifiesd in general medicine in the Alpes-Maritimes district. Gastroenterol Clin Biol 2003; 27: 37680 .

7. Riestra S, Fernández E, Leira P, García S, Ocio G, Rodrigo L. Prevalence of hepatitis $\mathrm{C}$ virus infection in the general population of northern spain. European Gastroenterol Hepatol 2002; 13 859: 447-81.

8. Sacristán B, Gastanares MI, Elena A, Sacristán M, Barcenilla J, García JC, et al. Seroepidemiologic study of hepatitis $C$ virus infection in a general population from the region of la Rioja, Spain. Medicina Clínica (Barc) 1998; 110 (2): 78.

9. El-Hazmi M. Prevalence of HBC, HCV, HIV-1,2 and HTLV-I/II infections among blood donors in a teaching hospital of the central region of Saudi Arabia. Saudi Med J 2004; 25: 26-33.

10. Fano R, Gonzales O, Jongres A, Hernández M. Prevalencia de anticuerpos contra el virus de la hepatitis $\mathrm{C}$ en un banco de sangre. Revista Cubana de Medicina Militar. 1995.

11. Dalgar O, Jeasson S, Skaug K, Rakerad N, Bell H. Hepatitis C in the general adult population of Oslo: prevalence and clinical spectrum. Scand J Gastroenterol 2003; 38: 864-70.

12. Sherriff L, Maynon-White R. A survey of hepatitis C prevalence amongst the homeless community of Oxford. J Public Health Med 2003; 25: 358-61.

13. Butterfield M, Bosworth H, Stechuchak K, Frothingham R, Bas L, Mmeador, et al. Racial differences in hepatitis B and hepatitis $\mathrm{C}$ and associated risk behaviors in veterans with severe mental illness. J Natl Med Assoc 2004; 96: 43-52.

14. Tadakata Yamada. Manual de Gastroenterología. Interamericana. México 1999: 678.

15. Simo R, Hernández C, Genesca J, Jardi R, Mesa J. High prevalence of hepatitis C virus infection in diabetic patients. Diabetes Care 1996; 19: 998-1000.

16. Heim A, Wagner D, Rothamel T, Hartmann U, Flik J, Verhagen W. Evaluation of serological screening of cadaveric sera for donors selection for cornea transplantion. J Med Virol 1999; 58: 291-5.

17. Zhang X, Constantine NT, Li L, Abdel-Hamid M, Bansal J, Smialek J. Application of comercial assays to detect IgG antibodies to hepatitis C virus in orine: a pilot study from autopsy cases. J Med Virol 1994; 44: 187-91.

18. Siragusa R, Passarino G, Mollo F, Tappero P. HVC positivity at postmortem among 793 heroin addicts examined in Piedmont (Italy). Panminerva Med 2001; 43: 119-21.

19. Khan T, Rizvi F, Rashid A. Hepatitis C seroposivity among chronic liver disease patients in hazera, Pakistan. J Ayub Med Coll Abbottabad 2003; 15 (2): 53-5

20. Brown R, Gaglio P. Scope of worldwide hepatitis C problem. Liver Transpl 2003; 9: S 10-3.

21. Zein N. The epidemiology and natural history of hepatitis $\mathrm{C}$ virus infection. Cleve clin J Med 2003; 70 (Supl. 4): S 2-6. 